\title{
Small Pixelled Antenna with MEMS-Reconfigurable Radiation Pattern
}

\author{
D. Rodrigo*(1), Y. Damgaci( ${ }^{(2)}$, M. Unlu ${ }^{(2)}$, L. Jofre $^{(1)}$ and B.A. Cetiner ${ }^{(2)}$ \\ (1) Universitat Politècnica de Catalunya, Signal Theory and Communications \\ Department, Barcelona 08034 Spain \\ (2) Utah State University, Electrical and Computer Engineering Department, \\ Logan, UT 84322, USA \\ E-mail: rodrigo@tsc.upc.edu
}

\section{Introduction}

To deliver robust and reliable communications, there has recently been significant interest in developing a single Multifunctional Reconfigurable Antenna (MRA) providing pattern reconfigurability with an emphasis on beam tilting that improves wireless communication capacity and bit error rates [1]. The design of an MRA with adaptive radiation pattern for mobile terminals is complex because of the usual tight constrains in the device maximum size. These restrictions typically prevent the use of multiple-antenna solutions such as phase arrays. Radiation pattern reconfigurability is usually achieved by the activation or deactivation of parasitic elements [2]. However, in order to preserve the antenna matching, it is typically required that a certain distance (of the order of one wavelength) between the active and parasitic parts is kept, which often exceeds the maximum allowed antenna size.

In this paper, a MEMS-switched small monopole antenna with reconfigurable radiation pattern is presented (Fig.1). It is designed to cover the U-NII-2E and UNII-3 bands that span from $5.47 \mathrm{GHz}$ to $5.825 \mathrm{GHz}$ and its size is roughly $\lambda / 2 \mathrm{x}$ $\lambda / 2$ (Fig.2), which is compatible with usual sizes of cellular phones and other personal mobile terminals.

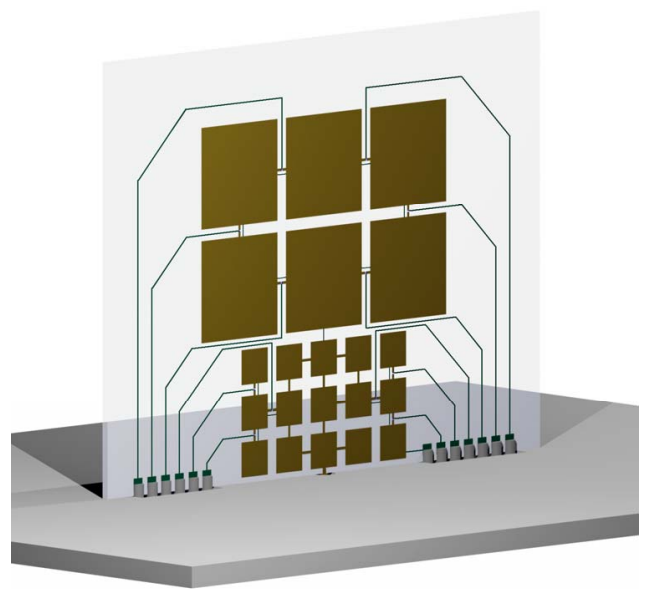

Fig. 1. Artist view of the MRA monopole, including biasing lines.

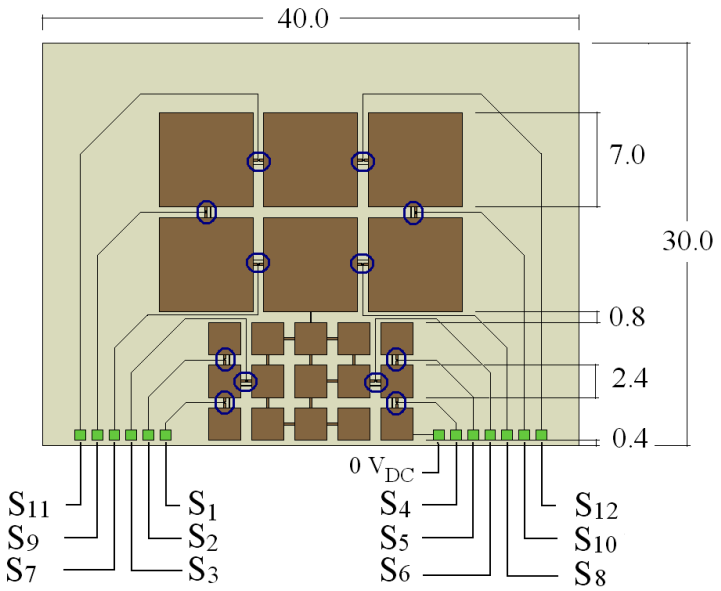

Fig. 2. Detailed sketch specifying dimensions and switch enumeration. 


\section{Reconfigurable antenna}

The antenna consists in a switched grid of electrically small metallic patches $[3,4]$. The placement of a switch at several patches adjacencies permits the modification of the current distribution over the antenna and thus modify the direction of maximum radiation.

\subsection{Antenna geometry}

The pixelled surface of the antenna has been split in two regions. The lower region which is near to the RF input port is responsible of the antenna matching, while the upper region allows the modification of the radiation pattern. In order to minimize the influence of the upper part over the input impedance, this region is left as a parasitic area. This isolation of functionalities allows an independent optimization of the pixel size for each region. Since the input impedance is more sensitive to individual pixel dimensions than radiation diagram, a finer grid of patches has been designed for the lower region.

In fact, this geometry employs the parasitic elements technique discussed in the first section. However, the addition of the small pixel area acts as a matching network and permits the location of the parasitic zone much closer to the feeding point, thus reducing the antenna size.

\subsection{Switching mechanism}

The connections between adjacent pixels of the antenna are by DC-contact MEMS switch with cantilever design. This switch structure is marked by high isolation, simple mechanical structure and relative robustness to the residual stress. In the present design, cantilevers are $115 \mu \mathrm{m}$ long and $80 \mu \mathrm{m}$ wide and are going to be fabricated using $5 \mu \mathrm{m}$-thick electroplated gold. The pull-down electrodes have an actuation area of $80 \times 80 \mu \mathrm{m}^{2}$ and the gap height is $1.4 \mu \mathrm{m}$.

The contact is provided by 4 dimples each with lateral dimension of $5 \mu \mathrm{m}$ by $5 \mu \mathrm{m}$ and $0.4 \mu \mathrm{m}$ thickness. This choice of dimensions results a pulldown voltage of $39 \mathrm{~V}$ as revealed by Coventorware mechanical simulations. The extracted simulation results of switch resistance $R_{s}$ and up-state capacitance $\mathrm{C}_{\text {up }}$, are 1-2 ohm and 14$16 \mathrm{fF}$, respectively. Figure 3 shows a conceptual view of the MEMS switch.

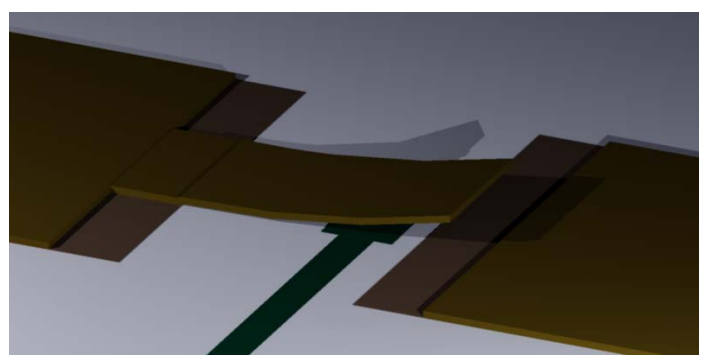

Fig. 3. MEMS switch conceptual view. Cantilever vertical displacement is magnified for visualization reasons.

In order to determine the position of the switches, we analyzed the contribution of each switch to the antenna reconfigurability by examining each switch status variability when a fully switched model is optimized to synthesize different radiation patterns. This criterion has been made compatible with the avoidance of 
vias in the biasing lines. These lines have been designed to cross the ground plane in order to place the biasing circuitry below it, which also reduces their coupling with the antenna radiating structure.

\section{Simulation and Results}

The reconfigurable antenna has been optimized for the maximization of the gain (including mismatch losses) in a certain radiation direction. Since the antenna has a planar geometry, directions of arrival (DOA) have been chosen over the antenna plane. The procedure for computing a suitable configuration has been based on rigorous EM full-wave analyses used jointly with a genetic optimization algorithm. The full-wave analysis has included the exact MEMS geometry, and the uncoupling of the bias lines has been checked.

The different angles of arrival and the corresponding switch configurations are summarized in Table I. The radiation pattern and return loss of each configuration is presented respectively in Fig. 4 and Fig. 5. These results demonstrate a good steering capability, changing the main beam direction in a range of around $120^{\circ}$ while maintaining a good matching over the whole frequency range.

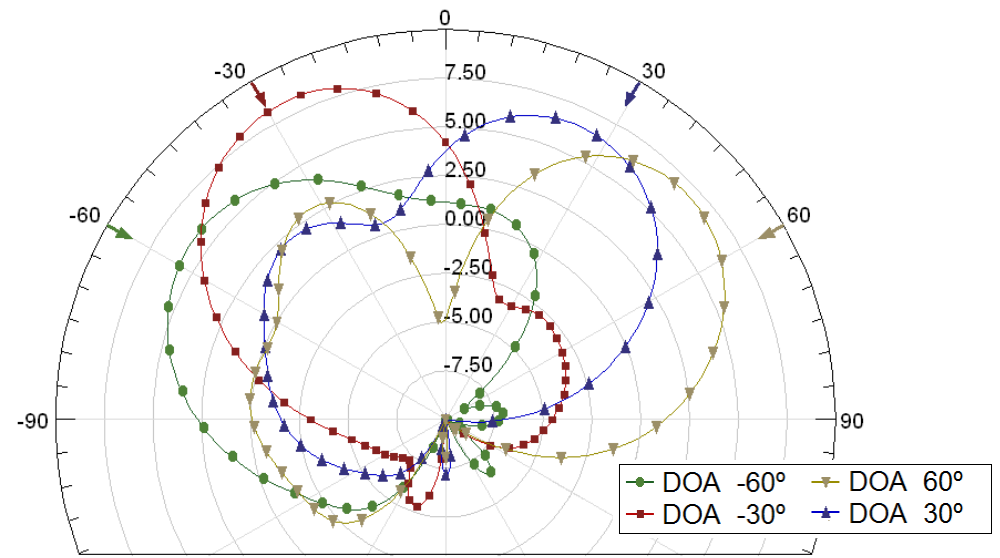

Fig. 4. Directivity radiation pattern of each MRA configuration [dB]

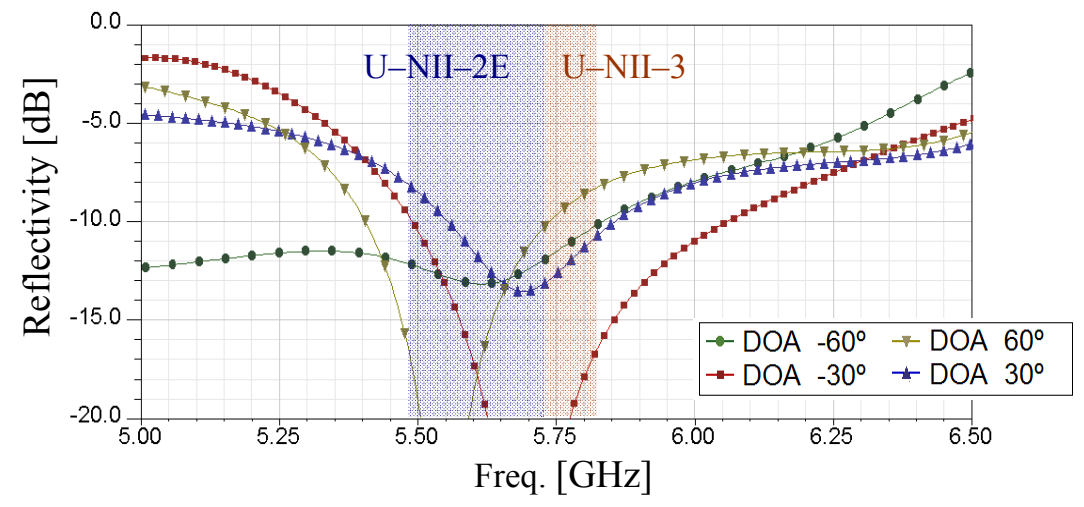

Fig. 5. Reflectivity of each MRA configuration 


\begin{tabular}{cccccccccccccc}
\hline & & $\mathrm{S}_{1}$ & $\mathrm{~S}_{2}$ & $\mathrm{~S}_{3}$ & $\mathrm{~S}_{4}$ & $\mathrm{~S}_{5}$ & $\mathrm{~S}_{6}$ & $\mathrm{~S}_{7}$ & $\mathrm{~S}_{8}$ & $\mathrm{~S}_{9}$ & $\mathrm{~S}_{10}$ & $\mathrm{~S}_{11}$ & $\mathrm{~S}_{12}$ \\
\hline DOA & $-60^{\circ}$ & 0 & 0 & 1 & 0 & 0 & 1 & 0 & 0 & 1 & 0 & 0 & 1 \\
DOA & $-30^{\circ}$ & 1 & 0 & 0 & 0 & 1 & 1 & 1 & 0 & 1 & 1 & 0 & 1 \\
DOA & $30^{\circ}$ & 0 & 1 & 0 & 1 & 0 & 1 & 0 & 0 & 1 & 1 & 1 & 0 \\
DOA & $60^{\circ}$ & 0 & 1 & 0 & 0 & 1 & 1 & 0 & 1 & 1 & 1 & 1 & 0 \\
\hline
\end{tabular}

Table I: Switch Configurations

\section{Conclusions}

A MEMS-reconfigurable antenna capable of steer the main beam over a wide range of angles has been designed. The use of a novel multisize pixelled geometry combined with the parasitic elements modification technique has lead to a structure with a low number of MEMS-switches. It has been achieved a small size antenna $(\lambda / 2 \times \lambda / 2)$ which can make possible its integration in mobile terminals allowing robust communications

\section{Acknowledgment}

This work was supported in part by the Spanish Interministerial Commission on Science and Technology (CICYT) under projects TEC2007-66698-C04-01/TCM and CONSOLIDER CSD2008-00068, by the Universitat Politècnica de Catalunya through the FPI-UPC Grant program, and by the United States National Institute of Justice under grant 2009-SQ-B9-K005.

\section{References}

[1] C. P. Sukumar, H. Elsami, A. Eltawil, and B. A. Cetiner, "Link Performance Improvement using Reconfigurable Multi-Antenna Systems," IEEE Antennas and Wireless Propagation Letters, Vol. 8, pp: 873 - 876, 2009

[2] G. H. Huff and J. T. Bernard, "Reconfigurable Antennas", in Modern Antenna Handbook, C.A. Balanis, Ed. New York: Wiley, 2008

[3] A. Grau, J. Romeu, L. Jofre, and F. De Flaviis. "A software defined MEMSreconfigurable pixel-antenna for narrowband MIMO systems," In Adaptive Hardware and Systems, 2008. AHS '08. NASA/ESA Conference on, pp: 141-146, June 2008

[4] L.N. Pringle et al., "A reconfigurable aperture antenna based on switched links between electrically small metallic patches", Antennas and Propagation, IEEE Transactions on, 52(6): 1434-1445, June 2004 